\title{
Biorelevant Dissolution Testing of Drug-Eluting Stents: Experiences with a Modified Flow-Through Cell Setup
}

\author{
Anne Seidlitz' ${ }^{1}$, Stefan Nagel', Beatrice Semmling ${ }^{1}$, \\ Niels Grabow ${ }^{2}$, Katrin Sternberg ${ }^{2}$, and Werner Weitschies ${ }^{1, *}$ \\ 'Institute of Pharmacy, Biopharmaceutics, and Pharmaceutical Technology, EMA University of \\ Greifswald, Friedrich-Ludwig-Jahn-Strasse 17, 17487 Greifswald, Germany \\ 2Institute for Biomedical Engineering, University of Rostock, 18119 Rostock, Germany
}

\begin{abstract}
In vitro dissolution testing of drug-eluting stents (DES) poses a special challenge in terms of apparatus design due to the very specialized local treatment of the vessel wall in the immediate vicinity of the blood flowing through the vessel and the stent lumen. A vessel-simulating flow-through cell was designed to emulate the placement of a DES in vivo and the flow through the lumen in a simplified in vitro setup, which also allows for the examination of distribution processes. The method is based on the compendial flow-through cell apparatus (USP 4), which was modified by the addition of a hydrogel compartment that represents the vessel wall and the adaptation of the flow rate to the blood flow rate in the coronary vessels. A comparison of the dissolution and release results obtained with the vessel-simulating flow-through cell with standard paddle (USP 2) and flow-through cell (USP 4) apparatus methods shows that release from the coating was decelerated by embedding in the hydrogel in the adapted apparatus. Further experiments with both hydrophilic and hydrophobic fluorescent model compounds coated onto the stents were performed to investigate the effects of different method parameters and variations in the coating composition. While release and distribution of hydrophilic fluorescein sodium were dependent on the flow rate and implantation technique, release kinetics of hydrophobic triamterene were influenced by the coating thickness and the model substance content of the coating. These results illustrate the importance of choosing the correct apparatus design and test parameters adapted to biorelevant conditions for specialized dosage forms such as DES.
\end{abstract}

\section{INTRODUCTION}

$\mathrm{D}$ rug-eluting stents (DES) are medical devices designed to restore blood flow after implantation into stenotic blood vessels and prevent reocclusion of the stented vessel (in-stent restenosis) caused by intimal hyperplasia by releasing suitable drug substances over prolonged periods of time. Stents typically consist of metallic backbones (bare-metal stent, BMS) coated with biodegradable or biostable homopolymers, copolymers, or polymeric blends, into which the active ingredients are incorporated. The marketed drug substances contained in currently marketed DES are mostly antiproliferative agents, such as paclitaxel and sirolimus (also known as rapamycin) and analogues.

Despite the tremendous success of DES in reducing the target lesion revascularization rate $(1,2)$ and their millionfold clinical usage since the first approval in 2002, many questions regarding the in vivo release profiles remain unanswered (3). While studies with different DES formulations favored the slow-release coatings $(4,5)$, the success of drug-eluting balloons suggests that short

*Corresponding author. contact times during which larger amounts of drug are delivered may also be suitable to prevent restenosis (6). In vivo determination of release profiles of DES is hampered by the fact that the released amounts of drug are typically very small. This means that plasma drug levels can typically only be monitored during the first phase of release, if at all. For the sirolimus-eluting Cyper stent (Johnsons \& Johnson, Inc.), which is supposed to release drug over 90 days according to the manufacturer's specification, detectable plasma concentrations have been reported for the first seven days (7). In another published report for the everolimus-eluting XIENCE V (Abbott Vascular), drug concentration in the plasma could be determined only over the first $72 \mathrm{~h}$ (8) before dropping below the limit of quantification; yet complete release from the device takes 120 days (9). However, drug release from DES is designed to be a topical application to the stented portion of the vessel wall, and drug dissolving in the blood is most likely cleared from the site at which it should prevent restenosis. Therefore, even if the plasma levels can be determined, the concentrations measured are not necessarily representative of the concentrations at the site of action (10). To fully characterize in vivo release from stents, the determination of tissue concentrations 
and residual content of the stent coatings at different time points is necessary. Such studies, even though laborious, are feasible in animal models. However, the prediction of device efficacy is not reliable based on the currently used animal models (11) because distinct differences between the response of humans and animal models have been reported $(3,12)$.

Because of these difficulties in determining in vivo release from DES, an in depth in vitro characterization of the performance of the devices under biorelevant testing conditions is crucially important for this dosage form.

There have been numerous discussions on dissolution testing of controlled-release parental products and the lack of standardized compendial methods (13-15). Possible applicable compendial methods for dissolution testing of DES include the paddle apparatus (USP 2), the flow-through cell (USP 4), and the reciprocating holder (USP 7) (16) equipped with a specialized stent holder, although there are few published reports of the usage of these methods (17-19). For DES, mostly noncompendial incubation methods have been reported (20-24). A detailed description of the use of these different methods has been reported (25).

The absence of regulatory guidance regarding a suitable dissolution test setup for controlled-release parenterals may be, in part, because the dosage forms as well as the sites of application are very diverse. For DES, the dosage form is in contact with two dissimilar acceptor compartments, the blood and the vessel wall. The blood flows past the implant in vast amounts depending on blood flow velocity and vessel diameter. Blood flow rates of approximately $35 \mathrm{~mL} / \mathrm{min}$ have been reported for coronary arteries (26), providing rapid clearance of drug released into the blood from the site of implantation. Because of the changes in vessel wall morphology with disease progression, the placement of the stent inside the vessel, and possibly tissue prolapse between the stent struts (27), it is difficult to predict the hydrodynamics inside the vessel. On the other hand, the vessel wall consists of different layers of cells including the intima, medium, and adventitia. Since the vessel wall is an "unstirred" compartment, diffusion layers and large concentration gradients may possibly arise locally at times of high release rates and influence release and distribution. Besides diffusion and convection, active transport proteins may play a role in the transport of dissolved drug in the vessel wall. Furthermore, the local distribution within the tissue as well as the distribution between the vessel wall and the blood will be influenced by specific binding proteins, which have been identified for both paclitaxel and sirolimus in the vessel wall (28). The constitution of the cell layers may change with disease progression, including the formation of lipid plaques and calcifications, and with the inflammatory reaction caused by stent implantation. These changes in cell constitution may also influence drug distribution (29).
(A)
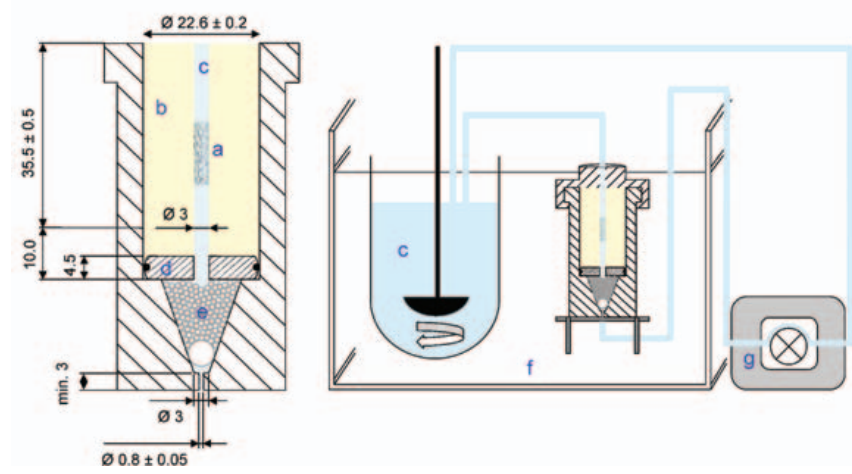

Figure 1. Schematic of (A) the vessel-simulating flow-through cell and $(B)$ the complete experimental setup with (a) stent, (b) hydrogel, (c) medium, (d) acrylic glass disc, (e) glass beads, (f) water bath, and $(\mathrm{g})$ peristaltic pump (dimensions in $\mathrm{mm}$ ).

With many incompletely understood parameters and inter- and intraindividual variations in the conditions at the site of implantation, it is obviously complicated, if not impossible, to accurately model all in vivo parameters. However, the precise modeling of all in vivo parameters is not the goal of designing biorelevant dissolution test systems. Rather, such test systems should include controlled and standardized conditions that represent the key aspects of the in vivo situation and have a distinct influence on the release profile. The need to include some biorelevant features in a dissolution test for DES has also been recognized by the United States FDA, which published a draft guidance (30) in 2008 stating that the in vitro release from DES should be examined under appropriate conditions based on the release mechanism and emulating hydrodynamic considerations of stent deployment.

\section{APPARATUS DESIGN AND EXPERIMENTAL CONDITIONS}

As described above, upon implantation DES are in contact with two dissimilar acceptor compartments; the outer (abluminal) side of the coating is pressed against the mechanically stable vessel wall, whereas the inside of the stent including the luminal coating is in contact with the free-flowing blood. From this positioning, differences in the release from the different sides of the coating may arise. The vessel-simulating flow-through cell (Figure 1) was designed to account for this positioning of the implanted stent in an in vitro setup. On one hand, it is possible to create flow conditions resembling arterial blood flow in this apparatus. On the other hand, a compartment that acts as a simplistic simulation of the vessel wall can be added. Since this compartment had to be diffusible for the released substances but mechanically stable, a hydrogel was used for this purpose. Calcium alginate was chosen as the gelling agent because 
of its stability at body temperature (31), the feasibility to adapt elasticity and gel strength as necessary (32), as well as its mild gelling conditions. These enable the incorporation of substances that are very sensitive to the surroundings, such as proteins or living cells (33). Such properties may be valuable in future studies when attempting to adapt the gel properties, such as lipophilicity, or availability of binding proteins to the properties of the vessel wall in vivo.

As described previously (34), the flow-through cell apparatus (USP 4) equipped with the standard tablet cell (diameter $22.6 \mathrm{~mm}$ ) was chosen as the basis, simulating release from stents into the blood and the vessel wall. For this purpose, the cylindrical section was elongated by $10 \mathrm{~mm}$, creating a shoulder onto which an acrylic glass disc with a central thread was placed. A stainless steel placeholder rod (diameter $3 \mathrm{~mm}$ ) was screwed into the thread. The calcium alginate gel was prepared using an internal gelation method, poured into the cell, and allowed to set before the central placeholder rod was removed, thus creating an opening in the hydrogel allowing for stent implantation and subsequent perfusion. A stent was implanted into the prepared cell by means of a balloon catheter and a catheter pump ( $6 \mathrm{~atm} / 15 \mathrm{~s})$, and the closed cell was placed in a water bath at $37^{\circ} \mathrm{C}$. Immediately following implantation, the opening in the hydrogel and the stent lumen were perfused with $\mathrm{pH} 7.4$ phosphate buffered saline Ph. Eur. (PBS) at a flow rate of $35 \mathrm{~mL} / \mathrm{min}$, which corresponds to the blood flow rate in the coronary vessels (26). The system was operated in a closed loop, and samples were drawn from the stirred, constant temperature $\left(37^{\circ} \mathrm{C}\right)$ medium container at predetermined times. Operating the system in an open loop would have been more representative of the situation in vivo where the drug is usually cleared from the circulating blood. This was not possible, though, because of the high flow rates and resulting analytical limitations. At the end of the experiment, the stent was removed from the gel, and the stent as well as the hydrogel were analyzed for drug content.

The equilibrium distributions at the end of the experiments are not necessarily representative of in vivo distributions, but rather reflect the physicochemical properties of the different compartments and released substances, as well as the recirculation of the dissolved drug. To examine the distribution processes at earlier times (e.g., between hydrogel and medium), experiments can be discontinued at different, predetermined time points. Alternatively, the spatial distribution in the hydrogel can be examined using the vessel-simulating flow-through cell if microscopically detectable model substances, such as fluorescent dyes, are released from the stent. A detailed description of how alginate cross-sections and alginate films lining the lumen can be prepared and examined is given elsewhere (35). Using these techniques, distribution depth and spatial distribution in relation to the distance from the areas of stent contact can be visualized.
The dissolution experiments described here were conducted using stents that were coated with fluorescent model substances with very different physicochemical properties. Fluorescein sodium, which is freely soluble in water (0.1-1.0 g/mL) (36) and has a log $P$ value of -1.52 (37), was chosen as a hydrophilic compound. Triamterene, a potassium-sparing diuretic with water solubility of $28 \mu \mathrm{g} / \mathrm{mL}$ (38) and $\log P$ value of 1.25 (39), was used as a fluorescent hydrophobic model compound. The fluorescent model substances were embedded in a blend of ammonio methacrylate copolymer type $A$ and $B$ (Eudragit RL/Eudragit RS, 3:7 ratio). The coating was performed by either dip- or spray-coating. For dip coating, the stents were mounted on a mandrel and dipped into the polymer solution containing the dissolved or suspended model substance. Dip coating is a very fast and easy process during which little loss of coating liquid occurs. However, the resulting coating layer mass is determined by the viscosity of the dipping solution. The viscosity must be adapted to allow for the desired coating layer mass while assuring homogeneous coatings without coating defects, such as coating bridges between the struts. Because of this limitation, a spray-coating process was performed to obtain homogeneously coated stents of very different coating layer masses. Spray-coating of single stents is much more time consuming, requires specialized equipment, and is associated with greater loss of coating liquid. The coating layer mass can easily be controlled by adjusting the process time. Since the coating process may influence release, as previously reported (40), a comparison of release from stents with similar drug loadings but coated with the two different processes was performed before further experiments were conducted. This comparison yielded no differences between the dip-coated and spray-coated DES (data not shown). Unless stated otherwise, the drug content amounted to $20-25 \%$ of total solid coating content. The approximate coating mass was determined by weighing. The loading was calculated based on the mass gain and the model substance content of the coating. The medium volume was adapted to the theoretical loading to assure sink conditions throughout the experiment $(250 \mathrm{~mL}$ or $500 \mathrm{~mL})$. Model substance content of samples was determined fluorimetrically using a microplate reader (Fluoroskan II, Labsystems, Helsinki, Finland or Varioskan, Thermo Fisher Scientific Inc., Waltham, MA, USA). After removal, the stents were analyzed for residual coating content by subsequent elution into PBS and fluorimetric measurement as described above until no further release within $48 \mathrm{~h}$ was detectable.

Hydrogels were liquefied by the addition of 10-fold concentrated $\mathrm{pH} 7.4$ phosphate buffer USP and transferred to microplates, and the fluorescence of the samples was measured. Calibration was performed with every experiment using a standard solution of the model substance in the respective sample matrix. For all quantitative measurements, the model substance content of two identical samples was determined, and the mean 
was used for further calculations. Reduction of medium volume from sampling and evaporation was accounted for in the calculations. Results (means \pm SD of $n=6$ or $n=3$ separate experiments as indicated in the figure captions) are presented in percentage of the sum of the amounts detected in all three compartments (medium, hydrogel, and stent coating) at the end of the experiment.

To evaluate whether the employed embedding and flow conditions in the vessel-simulating flow-through cell have an influence on release, release testing was also performed using a paddle (USP 2) and an unmodified flow-through cell (USP 4) apparatus. In these cases, the pre-expanded DES was placed directly inside the vessel or on top of the glass beads without further positioning. The agitation was set to $50 \mathrm{rpm}$ in the USP Apparatus 2, and the flow rate was set to $35 \mathrm{~mL} / \mathrm{min}$ in the flow-through cell. In another set of experiments, the influence of the flow rate on release in the vessel-simulating flow-through cell was examined by comparing the flow rate of $35 \mathrm{~mL} / \mathrm{min}$ to the lowest standard compendial flow rate of $4 \mathrm{~mL} / \mathrm{min}$.

Furthermore, the influence of the coating thickness and the model substance content of the coating on release were determined.To obtain different coating thicknesses, the stents were spray-coated until the desired gain in mass was achieved. Coating layer thickness was calculated using the surface area of the stent, coating mass, and polymer density. In a different set of experiments, the release from stents containing different amounts of model substance in the coating $(10 \%, 20 \%$, or $50 \%$, calculated as percentage of total solid content) was compared. To assure that observed differences were not caused by altering coating thicknesses, it was necessary to coat stents with approximately the same coating layer mass independent of model substance content. The stents for these experiments were dip-coated. Since the average gain in mass during dip coating was influenced by the model substance fraction incorporated into the dipping liquid, the viscosity of the dipping liquid was adjusted by further addition of the solvent. Comparable gains in mass were achieved by using this technique for coatings containing $10 \%, 20 \%$, or $50 \%$ of model substance.

In the last set of experiments, the tubing system at the cell outlet was adapted with a hemostatic valve (Y-connector with rotating adapter and Tuohy-Borst valve (B. Braun Melsungen AG, Melsungen, Germany), which is also used during clinical stent implantation. This modification allowed for the implantation of the tested DES into the vessel-simulating flow-through cell under flow conditions while preventing medium loss at the site of catheter entry. The catheter with the mounted stent was thus introduced into the medium flowing at $35 \mathrm{~mL} / \mathrm{min}$. The time from first medium contact of the DES to balloon expansion was set to $1 \mathrm{~min}$. During expansion (15 s) of the stent, medium flow was discontinued due to the complete closure of the lumen by the balloon, which also occurs in vivo. Release and distribution between the different

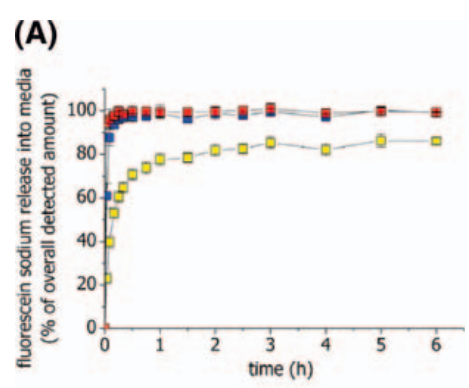

(B)

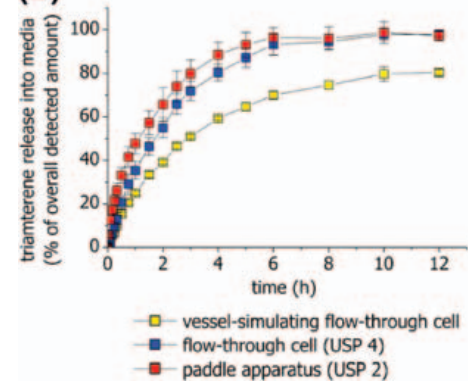

Figure 2. Normalized release of (A) fluorescein sodium and (B) triamterene from stent coatings into media over time with vessel-simulating flow-through cell, flow-through cell (USP 4), or paddle apparatus (USP 2); flow rate $35 \mathrm{~mL} / \mathrm{min}$, paddle speed $50 \mathrm{rpm}(\mathrm{n}=6$, mean $\pm S D)$. Adapted with permission from ref 34. Copyright 2008 Elsevier B. $V$.

compartments using this implantation technique were compared with implantation without media present in the lumen of the hydrogel. During these experiments, medium flow through the cell was initiated immediately after stent expansion and balloon withdrawal.

\section{RESULTS OF DISSOLUTION TESTING WITH THE VESSEL-SIMULATING FLOW-THROUGH CELL}

As reported previously (34), placement in the vesselsimulating flow-through cell had a great impact on release from the DES, resulting in a markedly slower release compared with the paddle (USP 2) and flow-through cell (USP 4) apparatus. As depicted in Figure 2, fluorescein sodium and triamterene release into the media was lower compared with the standard compendial methods. These data were then compared with an additional set of experiments with the vessel-simulating flow-through cell, during which the experiments were discontinued at predetermined times and the distribution in the three compartments medium, hydrogel, and stent coating was determined (data not shown, see (34)). The results reveal that the modification of the flow-through cell not only changed the release into the medium due to distribution of a fraction of the released substance into the hydrogel, but also directly changed the release rate from the coating. Since the coating composition was not altered, this change in release rate must be attributed to the positioning of the stent in the vessel-simulating flow-through cell in which the abluminal side of the struts 
(A)

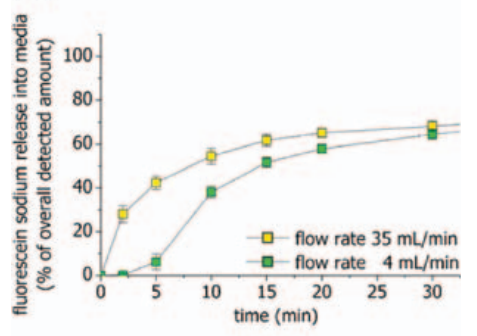

(B)

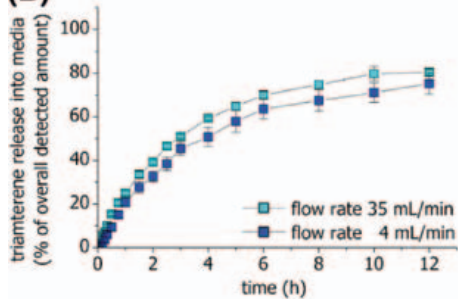

Figure 3. Normalized release of (A) fluorescein sodium and $(B)$ triamterene from stent coatings into media over time with the vessel-simulating flow-through cell at flow rates of $35 \mathrm{~mL} / \mathrm{min}$ or $4 \mathrm{~mL} / \mathrm{min}$ $(\mathrm{n}=6$, mean $\pm S D)$.

is not surrounded by free-flowing medium but is in direct contact with the hydrogel. This represents an "unstirred" compartment with different characteristics of drug transport. In this compartment, diffusion of small molecules is not hindered but drug transport through convection is minimized. The results emphasize the need to use specialized test systems for very specialized dosage forms, such as DES, which take the situation at the site of application into account. In the vessel-simulating flow-through cell, the in vivo situation is emulated with respect to appropriate stent embedding and flow conditions.

At this point, it should also be mentioned that DES testing in the compendial apparatus was associated with dislocations of the dosage form, which may possibly influence release. Constant movement of the dosage form in the vessel occurred in the paddle apparatus (USP 2), resulting in nonstandardized additional mechanical stress caused by numerous collisions of the DES with the apparatus. A reversible initial flotation due to gas bubbles trapped inside the stent was observed in the flow-through cell (USP 4). Even though the stents did not float on top of the medium throughout the experiment in either setup, it may be advisable to standardize the positioning of the device (e.g., by using a special sinker). In the vesselsimulating flow-through cell, the positioning of the stent is predetermined by the apparatus design and is therefore not variable.

The release into the medium at the flow rates of 4 and $35 \mathrm{~mL} / \mathrm{min}$ is depicted in Figure 3. For triamterene, the detected concentrations at both flow rates were very similar over the duration of the experiment. Release with the higher flow rate was slightly faster, but the difference
(A)

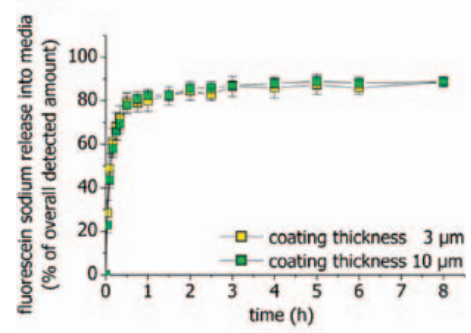

(B)

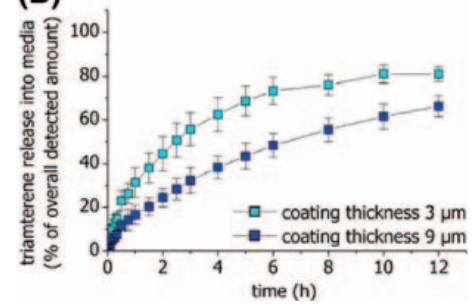

Figure 4. Normalized release of (A) fluorescein sodium and (B) triamterene from stent coatings of $3 \mu \mathrm{m}$ or 10/9 $\mu \mathrm{m}$ thickness into media over time with the vessel-simulating flow-through cell $(\mathrm{n}=6$, mean $\pm S D)$.

between two corresponding medium fractions did not exceed $5 \%$. This indicates that for triamterene, drug solubility is important for drug release. For fluorescein sodium, distinct differences were detectable within the first phase of release. After approximately $30 \mathrm{~min}$, the fractions released into the medium reached similar values, which were also obtained throughout the rest of the experiment (data not shown). The initial slower release into the medium for the lower flow rate can be caused by a deceleration of release from the coating, the accumulation of a larger fraction of the released substance in the hydrogel, or by a combination of the two processes. Assuming a diffusion layer model, the removal of the released substance at the boundary layer will be slower for the flow rate of $4 \mathrm{~mL} / \mathrm{min}$, resulting in smaller concentration gradients that will decelerate release into the medium. The same situation may arise for the redistribution from the hydrogel into the medium. Furthermore, fluorescein sodium transport to the medium container from which the samples are taken is slower when a lower flow rate is used. However, correction of the time points for this short delay only results in minor changes in the graph and does not account for the changes in medium fraction observed with the two different flow rates (data not shown).

The results of release testing for stents of different coating thicknesses are shown in Figure 4. The calculated coating thickness was $3 \mu \mathrm{m}$ for the thinner coating and $10 \mu \mathrm{m}$ (fluorescein sodium) or $9 \mu \mathrm{m}$ (triamterene) for the thicker coatings. For fluorescein sodium, no distinct deviations in the normalized release into the medium were observed in spite of the threefold increase in coating thickness. For triamterene, the amount released into the 
(A)

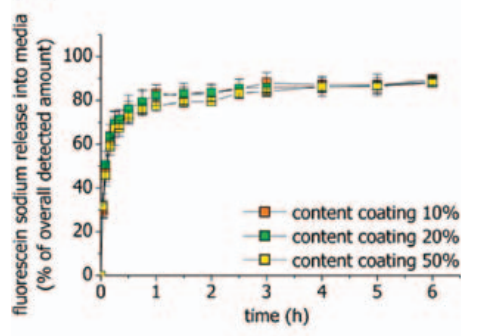

(B)

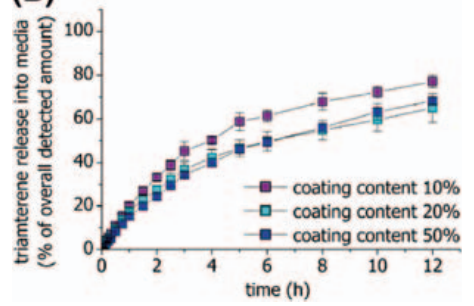

Figure 5. Normalized release of (A) fluorescein sodium and $(B)$ triamterene from stent coatings into media over time with the vessel-simulating flow-through cell as a function of model substance coating content $(10 \%, 20 \%$, or $50 \%) ;(\mathrm{n}=6$, mean $\pm S D)$.

medium, calculated as a percentage of the sum of the amounts detected in all 3 compartments at the end of the experiments, was smaller for the thicker coatings. In addition, the residual triamterene fraction in the coating at the end of the experiments (after $12 \mathrm{~h}$ ) was different for the two coatings, with $28 \%$ remaining in the $9-\mu$ m coating and $11 \%$ in the $3-\mu \mathrm{m}$ coating. This change in release rate may be attributed to an increase in diffusion pathway with increasing coating thickness. A plot of the absolute release into the medium for both coatings shows similar initial releases (data not shown), which is caused by the similar surface area and surface near drug load. The triamterene data agrees well with published experimental (40) and modeled (41) data for other compounds. For fluorescein sodium, the polymer does not seem to control the release rate resulting in comparable releases independent of coating thickness. These results are consistent with the finding that fluorescein sodium release was influenced by the flow rate, whereas triamterene release was unaffected by this parameter.

The results of the comparison of release into the medium from stents containing $10 \%, 20 \%$, or $50 \%$ of model substance in the coatings (expressed as percentage of total solids content) are depicted in Figure 5. According to Sharkawi et al. (40), an increase in release rate with increasing model substance content is to be expected. The authors explain that this accelerated release is caused by the formation of pores as the model substance is released. An increase in release is also to be expected because of the larger amount of drug particles present at the surface of the coating. However, this was not the case in the experiments described here. For fluorescein sodium, no differences were observed in spite of very different

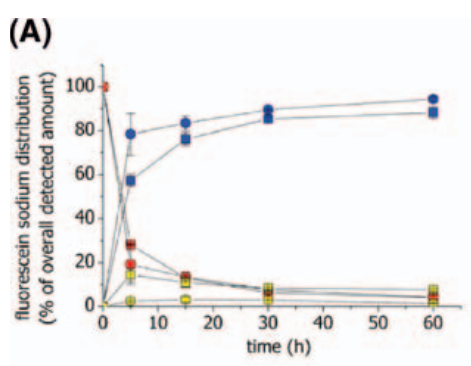

(B)
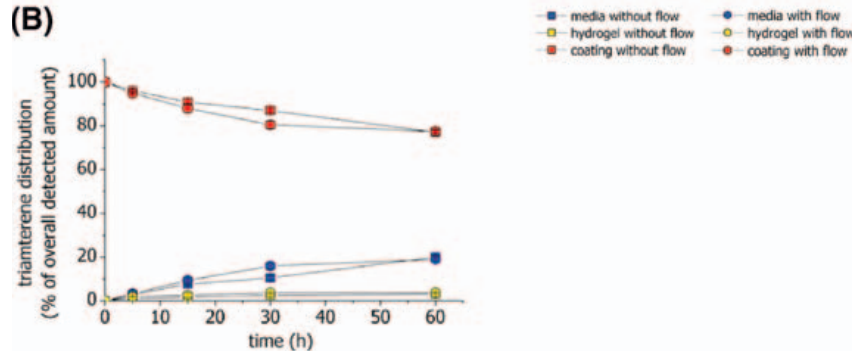

Figure 6. Normalized amount of (A) fluorescein sodium and (B) triamterene in the compartment medium, hydrogel, and stent coating over time in the vessel-simulating flow-through cell as a function of implantation technique, without medium present in the lumen or with medium flowing through hydrogel lumen during stent placement at $35 \mathrm{~mL} / \mathrm{min}(\mathrm{n}=3$, for each data point, mean $\pm S D$ ).

amounts of model substance. As already described for the different coating thicknesses, the applied polymer does not seem to control the release from fluorescein sodium-coated DES. For triamterene, releases into the medium from the coatings containing $20 \%$ or $50 \%$ of model substance were almost identical. The coating containing only $10 \%$ model substance showed a slightly different release that was, contradictory to the literature description, faster than that of the other two coating compositions. The amounts remaining in the coating after $12 \mathrm{~h}$ were quantified as 19\% (10\% model substance content), $27 \%$ ( $20 \%$ model substance content), and $29 \%$ (50\% model substance content). A possible explanation for the slower release from the coatings containing higher amounts of triamterene might be that the large amounts of the very hydrophobic model substance in the fairly thin coatings lead to hydrophobic interactions between the drug molecules, which could hinder release.

A comparison of the distribution of the model substance among the three compartments (i.e., medium, hydrogel, and coating) during the first $60 \mathrm{~min}$ as a function of the implantation technique (with flow through the hydrogel lumen or without flow medium present in the lumen during stent expansion) is shown in Figure 6. For fluorescein sodium, distinct differences in the initial fraction released into the hydrogel were detected and most likely result from the fast release of the hydrophilic compound. When implanting without flow, fluorescein sodium release from the stents starts immediately upon contact with the hydrogel, thus releasing an initial portion solely into the gel, since medium is not present during the first seconds. 
On the other hand, when implanting under flow, the coated stent is in contact with the medium first, and release into the medium starts before release into the hydrogel. For triamterene, which is released much slower from the coating, no differences in distribution were observed. Based on these experiments, the implantation technique can be expected to play a role for fast-releasing stent coatings or coatings with a high initial burst. In addition, this setup has great potential for usage in dissolution testing of drug-eluting balloons, which are designed to release drug within the short time of contact (approximately $1 \mathrm{~min}$ ) between the balloon and the vessel wall. To the authors' knowledge, no biorelevant testing system has been reported for this new generation of devices. The vessel-simulating flow-through cell with the modification of the hemostatic valve entry, which already includes the vessel wall representing compartment and the directed flow through an artificial lumen, seems a very suitable setup for this application.

\section{CONCLUSIONS}

The vessel-simulating flow-through cell enables dissolution testing of DES in a setup in which the embedding and flow conditions were designed to resemble the situation at the site of implantation in vivo. In this model, which is based on the compendial flow-through cell apparatus (USP 4), the vessel wall is represented by an additional hydrogel compartment. With the inclusion of this compartment, the examination of drug distribution became feasible.

A comparison with dissolution data obtained with two compendial methods (paddle, USP 2, and flow-through cell, USP 4, apparatus) shows that the release from the stent coating was influenced by the positioning and flow conditions present in the vessel-simulating flow-through cell. This finding emphasizes the necessity to include such parameters in dissolution testing of specialized dosage forms such as DES.

Evaluation of additional method parameters (flow rate and implantation technique) or coating variations (thickness and model substance content) resulted in very different outcomes for the hydrophilic and hydrophobic model substances included in the coatings. While the release and distribution of hydrophilic fluorescein sodium was susceptible to changes in flow rate and implantation procedure, changes in the coating had no impact on the release profiles. On the other hand, triamterene release was influenced by variations in coating thickness and model substance content, but neither the change in flow rate nor implantation technique had a distinct effect on release and distribution. This dissimilar response of the two model compounds to the variation in method and coating parameters of the two coating formulations underlines the need for case-by-case examination of different coating compositions and individual evaluations of their susceptibility to such changes. Taking the water solubilities and $\log P$ values of paclitaxel and sirolimus into account, their release from DES would be expected to be more similar to hydrophobic triamterene release than to release of hydrophilic fluorescein sodium.

With the addition of a hemostatic valve, the vesselsimulating flow-through cell allows for stent implantation under flow conditions, which may have a great influence on drug distribution for fast-releasing stent coatings. This setup may also be advantageous for dissolution testing of drug-eluting balloons.

\section{ACKNOWLEDGMENTS}

BMS as well as balloon catheters were generously provided by Biotronik SE \& Co. KG (Berlin, Germany). Hemostatic valves were kindly donated by B. Braun Melsungen AG (Melsungen, Germany). Eudragit RL and RS were gifts from Roehm GmbH (Darmstadt, Germany). This project was funded by the European Regional Development Fund (ERDF) and the European Social Fund (ESF) within the collaborative research between economy and science of the state Mecklenburg-Vorpommern and the Federal Ministry of Education and Research (BMBF) within REMEDIS.

\section{REFERENCES}

1. Moses, J.W.; Leon, M. B.; Popma, J. J.; Fitzgerald, P. J.; Holmes, D. R.; O'Shaughnessy, C.; Caputo, R. P.; Kereiakes, D. J.; Williams, D. O.; Teirstein, P. S.; Jaeger, J. L.; Kuntz, R. E. Sirolimus-eluting stents versus standard stents in patients with stenosis in a native coronary artery. New Engl. J. Med. 2003, 349 (14), 1315-1323.

2. Stone, G.W.; Ellis, S. G.; Cox, D. A.; Hermiller, J.; O'Shaughnessy, C.; Mann, J.T.; Turco, M.; Caputo, R.; Bergin, P.; Greenberg, J.; Popma, J. J.; Russell, M. E. A polymer-based, paclitaxel-eluting stent in patients with coronary artery disease. New Engl.J. Med. 2004, 350 (3), 221-231.

3. Venkatraman, S.; Boey, F. Release profiles in drugeluting stents: issues and uncertainties. J. Control. Release 2007, 120 (3), 149-160.

4. Lansky, A. J.; Costa, R. A.; Mintz, G. S.; Tsuchiya, Y.; Midei, M.; Cox, D. A.; O'Shaughnessy, C.; Applegate, R. A.; Cannon, L. A.; Mooney, M.; Farah, A.; Tannenbaum, M. A.; Yakubov, S.; Kereiakes, D. J.; Wong, S. C.; Kaplan, B.; Cristea, E.; Stone, G.W.; Leon, M. B.; Knopf, W.D.; O'Neill, W.W. Non-polymer-based paclitaxel-coated coronary stents for the treatment of patients with de novo coronary lesions: angiographic follow-up of the DELIVER clinical trial. Circulation 2004, 109 (16), 1948-1954.

5. Serruys, P.W.; Sianos, G.; Abizaid, A.; Aoki, J.; den Heijer, P.; Bonnier, H.; Smits, P.; McClean, D.; Verheye, S.; Belardi, J.; Condado, J.; Pieper, M.; Gambone, L.; Bressers, M.; Symons, J.; Sousa, E.; Litvack, F. The effect of variable 
dose and release kinetics on neointimal hyperplasia using a novel paclitaxel-eluting stent platform: the Paclitaxel In-Stent Controlled Elution Study (PISCES). J. Am. Coll. Cardiol. 2005, 46 (2), 253-260.

6. Scheller, B.; Hehrlein, C.; Bocksch, W.; Rutsch, W.; Haghi, D.; Dietz, U.; Bohm, M.; Speck, U. Treatment of coronary in-stent restenosis with a paclitaxel-coated balloon catheter. N. Engl. J. Med. 2006, 355 (20), 2113-2124.

7. Vetrovec, G.W.; Rizik, D.; Williard, C.; Snead, D.; Piotrovski, V.; Kopia, G. Sirolimus PK trial: a pharma cokinetic study of the sirolimus-eluting Bx velocity stent in patients with de novo coronary lesions. Catheter. Cardio. Inte. 2006, 67 (1), 32-37.

8. Wang, Q.; Pierson, W.; Sood, P.; Bol, C.; Cannon, L.; Gordon, P.; Saucedo, J.; Sudhir, K. Pharmacokinetic Sub-Study in the SPIRIT III Randomized and Controlled Trial of XIENCE V Everolimus Eluting Coronary Stent System. J. Interv. Cardiol. 2010, 23 (1), 26-32.

9. Perkins, L.E .L.; Boeke-Purkis, K. H.;Wang, Q.; Stringer, S. K.; Coleman, L. A. XIENCE V Everolimus-Eluting Coronary Stent System: A Preclinical Assessment. J. Interv. Cardiol. 2009, 22 (1), 28-40.

10. Kamath, K. R.; Barry, J. J.; Miller, K. M.The Taxus drug-eluting stent: a new paradigm in controlled drug delivery. Adv. Drug Deliv. Rev. 2006, 58 (3), 412-436.

11. Guideline on the Clinical and Non Clinical Evaluation During the Consultation Procedure on Medicinal Substances Contained in Drug-Eluting (Medicinal Substance-Eluting) Coronary Stents; EMEA/CHMP/ EWP/110540/2007; Committee for Medicinal Products for Human Use, European Medicines Agency: London, 2008.

12. Carter, A. J.; Aggarwal, M.; Kopia, G. A.; Tio, F.;Tsao, P. S.; Kolata, R.; Yeung, A. C.; Llanos, G.; Dooley, J.; Falotico, R. Long-term effects of polymer-based, slow-release, sirolimus-eluting stents in a porcine coronary model. Cardiovasc. Res. 2004; 63 (4), 617-624.

13. Siewert, M.; Dressman, J.; Brown, C. K.; Shah, V.P. FIP/ AAPS guidelines to dissolution/in vitro release testing of novel/special dosage forms. AAPS PharmSciTech 2003, 4 (1), E7.

14. Burgess, D. J.; Crommelin, D. J.; Hussain, A. S.; Chen, M. L. Assuring quality and performance of sustained and controlled release parenterals: EUFEPS workshop report. AAPS PharmSci 2004, 6 (1), E11.

15. Martinez, M.; Rathbone, M.; Burgess, D.; Huynh, M. In vitro and in vivo considerations associated with parenteral sustained release products: a review based upon information presented and points expressed at the 2007 Controlled Release Society Annual Meeting. J. Control. Release 2008, 129 (2), 79-87.

16. Parker, J.; Gray, V. Highlights of the AAPS workshop on dissolution testing for the 21 st century. Dissolution Technol. 2006, 13 (3), 26-31.

17. Rajender, G.; Narayanan, N. G. B. Liquid chromatography-tandem mass spectrometry method for determination of Sirolimus coated drug eluting nano porous carbon stents. Biomed. Chromatogr. 2010, 24, 329-334.
18. Merciadez, M.; Alquier, L.; Mehta, R.; Patel, A.;Wang, A. A novel method for the elution of sirolimus in drug eluting stents. Presented at the 39th Middle Atlantic Regional Meeting of the American Chemical Society, Collegeville, PA, May 16-18, 2007.

19. Kamberi, M.; Nayak, S.; Myo-Min, K.; Carter, T. P.; Hancock, L.; Feder, D. A novel accelerated in vitro release method for biodegradable coating of drug eluting stents: Insight to the drug release mechanisms. Eur.J.Pharm. Sci. 2009, 37 (3-4), 217-222.

20. Lockwood, N. A.; Hergenrother, R.W.; Patrick, L. M.; Stucke, S. M.; Steendam, R.; Pacheco, E.; Virmani, R.; Kolodgie, F. D.; Hubbard, B. In vitro and in vivo characterization of novel biodegradable polymers for application as drug-eluting stent coatings. J. Biomater. Sci.-Polym. E. 2010, 21 (4), 529-552.

21. Minghetti, P.; Cilurzo, F.; Selmin, F.; Casiraghi, A.; Grignani, A.; Montanari, L. Sculptured drug-eluting stent for the on-site delivery of tacrolimus. Eur. J. Pharm. Biopharm. 2009, 73 (3), 331-336.

22. Raval, A.; Parikh, J.; Engineer, C. Dexamethasone eluting biodegradable polymeric matrix coated stent for intravascular drug delivery. Chem. Eng. Res. Des. 2010, 88 (11), 1479-1484.

23. Guo, Q.; Knight, P.T.; Mather, P.T. Tailored drug release from biodegradable stent coatings based on hybrid polyurethanes. J. Control. Release 2009, 137 (3), 224-233.

24. Pan, C. J.;Tang, J. J.; Weng, Y. J.; Wang, J.; Huang, N. Preparation and in vitro release profiles of drug-eluting controlled biodegradable polymer coating stents. Colloid. Surface B 2009, 73 (2), 199-206.

25. Seidlitz, A.; Nagel, S.; Semmling, B.; Sternberg, K.; Kroemer, H. K.; Weitschies, W. In vitro dissolution testing of drug-eluting stents. Curr. Pharm. Biotechnol., in press.

26. Di Mario, C.; Meneveau, N.; Gil, R.; de Jaegere, P.; de Feyter, P. J.; Slager, C. J.; Roelandt, J. R.; Serruys, P.W. Maximal blood flow velocity in severe coronary stenoses measured with a Doppler guidewire. Limitations for the application of the continuity equation in the assessment of stenosis severity. Am. J. Cardiol. 1993, 71 (14), 54D-61D.

27. Jang, I. K.; Tearney, G.; Bouma, B. Visualization of tissue prolapse between coronary stent struts by optical coherence tomography: comparison with intravascular ultrasound. Circulation 2001, 104 (22), 2754.

28. Levin, A. D.; Vukmirovic, N.; Hwang, C.W.; Edelman, E. R. Specific binding to intracellular proteins determines arterial transport properties for rapamycin and paclitaxel. Proc. Natl. Acad. Sci. U.S.A. 2004, 101 (25), 9463-9467.

29. Tzafriri, A. R.; Vukmirovic, N.; Kolachalama, V. B.; Astafieva, I.; Edelman, E. R. Lesion complexity determines arterial drug distribution after local drug delivery. J. Control. Release 2010, 142 (3), 332-338. 
30. Coronary Drug-Eluting Stents-Nonclinical and Clinical Studies; Draft Guidance; U.S. Department of Health and Human Services, Food and Drug Administration, Center for Devices and Radiological Health (CDRH), U.S. Government Printing Office:Washington, DC, 2008. http://www.fda.gov/downloads/Drugs/Guidanc eComplianceRegulatoryInformation/Guidances/ UCM228704.pdf (accessed Oct 12, 2011).

31. Becker, T. A.; Kipke, D. R.; Brandon, T. Calcium alginate gel: a biocompatible and mechanically stable polymer for endovascular embolization. J. Biomed. Mater. Res. 2001,54 (1), 76-86.

32. Kuo, C. K.; Ma, P.X. lonically crosslinked alginate hydrogels as scaffolds for tissue engineering: part 1. Structure, gelation rate and mechanical properties. Biomaterials 2001,22 (6), 511-521.

33. Smidsrod, O.; Skjak-Braek, G. Alginate as immobilization matrix for cells. Trends Biotechnol. 1990, 8 (3), 71-87.

34. Neubert, A.; Sternberg, K.; Nagel, S.; Harder, C.; Schmitz, K. P.; Kroemer, H. K.;Weitschies, W. Development of a vessel-simulating flow-through cell method for the in vitro evaluation of release and distribution from drug-eluting stents. J. Control. Release 2008, 130 (1), 2-8.

35. Seidlitz, A.; Nagel, S.; Semmling, B.; Grabow, N.; Martin, H.; Senz, V.; Harder, C.; Sternberg, K.; Schmitz, K. P.; Kroemer, H. K.; Weitschies, W. Examination of drug release and distribution from drug-eluting stents with a vessel-simulating flow-through cell. Eur. J. Pharm. Biopharm. 2011, 78 (1), 36-48.
36. Fluorescein Sodium Monograph. In European Pharmacopoeia, 7th ed.; European Directorate for the Quality of Medicines, Council of Europe: Strasbourg, France, 2010.

37. Sakai, M.; Imai, T.; Ohtake, H.; Azuma, H.; Otagiri, M. Effects of absorption enhancers on the transport of model compounds in Caco-2 cell monolayers: assessment by confocal laser scanning microscopy. J. Pharm. Sci. 1997, 86 (7), 779-785.

38. Watanabe, E.; Takahashi, M.; Hayashi, M. A possibility to predict the absorbability of poorly water-soluble drugs in humans based on rat intestinal permeability assessed by an in vitro chamber method. Eur. J. Pharm. Biopharm. 2004, 58 (3), 659-665.

39. Zhao, Y.; Jona, J.; Chow, D. T.; Rong, H.; Semin, D.; Xia, X.; Zanon, R.; Spancake, C.; Maliski, E. High-throughput log $\mathrm{P}$ measurement using parallel liquid chromatography/ ultraviolet/mass spectrometry and sample-pooling. Rapid Commun. Mass Spectrom. 2002, 16 (16), 1548-1555.

40. Sharkawi, T.; Leyni-Barbaz, D.; Chikh, N.; McMullen, J. N. Evaluation of the in vitro drug release from resorbable biocompatible coatings for vascular stents. J. Bioact. Compat. Polym. 2005, 20 (2), 153-168.

41. Balakrishnan, B.; Dooley, J. F.; Kopia, G.; Edelman, E. R. Intravascular drug release kinetics dictate arterial drug deposition, retention, and distribution. J. Control. Release 2007, 123 (2), 100-108. 